\title{
The Childhood Obesity Research Demonstration Project: A Team Approach for Supporting a Multisite, Multisector Intervention
}

\author{
Nancy Williams, MSPH,', Carrie A. Dooyema, MPH, MSN, RN, \\ Jennifer L. Foltz, MD, MPH, ${ }^{1,2}$ Brook Belay, MD, MPH, and Heidi M. Blanck, PhD',2
}

\section{Abstract}

Background: Comprehensive multisector, multilevel approaches are needed to address childhood obesity. This article introduces the structure of a multidisciplinary team approach used to support and guide the multisite, multisector interventions implemented as part of the Childhood Obesity Research Demonstration (CORD) project. This article will describe the function, roles, and lessons learned from the CDC-CORD approach to project management.

Methods: The CORD project works across multisectors and multilevels in three demonstration communities. Working with principal investigators and their research teams who are engaging multiple stakeholder groups, including community organizations, schools and child care centers, health departments, and healthcare providers, can be a complex endeavor. To best support the community-based research project, scientific and programmatic expertise in a wide range of areas was required. The team was configured based on the skill sets needed to interact with the various levels of staff working with the project.

Conclusions: By thoughtful development of the team and processes, an efficient system for supporting the multisite, multisector intervention project sites was developed. The team approach will be formally evaluated at the end of the project period.

\section{Introduction}

$\mathbf{T}$ he prevention and reduction of childhood obesity is a public health priority. Results from the 2011-2012 National Health and Nutrition Examination Survey, using measured heights and weights, indicate that an estimated $16.9 \%$ of children and adolescents $2-19$ years of age are obese with higher rates among some subgroups, such as non-Hispanic black and Hispanic children. ${ }^{1}$

Because children with lower socioeconomic status may be at increased risk for obesity owing to a number of factors, ${ }^{2}$ there is a need to address childhood obesity in high-risk populations to improve health and reduce disparities. The Children's Health Insurance Program (CHIP) specifically enrolls children who have been identified based on family income. CHIP also enrolls many minority children and adolescents who are disproportionately burdened by obesity. ${ }^{3}$ Thus, obesity prevention efforts tar- geting the CHIP population have the potential to decrease obesity in an underserved population, as well as possibly reducing costs of related comorbidities.

The Institute of Medicine put forth a framework of the socioecological model (SEM) to address obesity that details the reciprocal relationship between individuals and their environments. ${ }^{4}$ This model has been adopted and has led to interventions that utilize multisetting, multilevel (MSML) approaches to support both children and their families and promote supportive environments in settings where children spend time, such as schools and child care centers. ${ }^{5}$ By connecting and reinforcing interventions in public health, healthcare, and the community at large, MSML approaches have the potential to assist all children and families in communities by addressing obesity through both prevention and treatment approaches in key settings. ${ }^{6-8}$ This MSML approach is supported by the SEM, ${ }^{6}$ which identifies the relationship between individuals and their environment,

'Division of Nutrition, Physical Activity, and Obesity, National Center for Chronic Disease Prevention and Health Promotion, Centers for Disease Control and Prevention, Atlanta, GA.

2United States Public Health Service Commissioned Corps, Atlanta, GA. 
including settings such as healthcare, schools, early care and education, and the community at large. As such, the SEM recognizes that individuals are influenced by relationships with others as well as other effectors of behavior, such as system-level effects and public policy factors. The MSML approach is also supported by the obesity chronic care model, ${ }^{9}$ which postulates that system-level changes within healthcare and other settings can reinforce individual selfmanagement of obesity-related behaviors.

\section{Methods}

The Childhood Obesity Research Demonstration (CORD) project is a cooperative agreement with four grantees funded and supported by the CDC. ${ }^{10}$ The CORD grant sought applications for interventions designed to address obesity among children 2-12 years of age who are eligible for Medicaid or CHIP or who live in low-income areas where $50 \%$ or more of students are eligible for the National School Lunch Program. The CORD grant offered 4 years of funding to three demonstration grantees to develop, implement, and evaluate unique MSML approaches. The grant also supported an additional grantee to design and implement an objective, rigorous evaluation of the overall project.

CORD uses a comprehensive model for reducing childhood obesity among underserved children, including those eligible for CHIP, and includes three demonstration sites and one evaluation center. The CDC-CORD communitybased interventions follow the MSML approach by spanning the SEM and including the home, school, early care and education, healthcare, and community settings, as well as individual, interpersonal, community, and system-wide levels. ${ }^{11}$ A unique aspect of CDC-CORD is to use the MSML approach to develop clinical-community linkages to support children and their families. In this model, healthcare providers and members of the team, such as community health workers, provide referrals to community resources and support for children and their families or even provide hands-on instruction on how to make healthier choices in the community.

As part of CDC-CORD, the CDC developed a team approach for monitoring and administering the cooperative agreement, as well as a system to promote collaboration and cooperation among the demonstration sites. This article describes the multidisciplinary management and administrative efforts designed to ensure the success of the CORD program and how this approach may be valuable in planning program administration for future public health efforts.

Team science has become an important area in translational research. ${ }^{12}$ Diverse teams have several values. They can accelerate innovation by creating new insight from the combined knowledge of the team as a whole. ${ }^{13}$ Diverse teams are not dominated by a single view, and their knowledge base and connections to information are more robust. Teams also help sustain innovation because the different team members make connections between different ideas and processes. Further details, including grantee information, creation of common measures, and timeline of the project, are described in other articles in this issue of Childhood Obesity.

\section{Team Formation and Initial Planning}

The process that the Division of Nutrition, Physical Activity, and Obesity used to form a team to oversee the CORD project was built on team science, as well as the realization that working with grantees and their community partners requires different levels of expertise. Working with principal investigators (PIs) and their research teams who are integrating many stakeholder groups, such as community organizations, health departments, and healthcare providers, can be a complex endeavor. Project officers (POs) are commonly used to help manage and oversee federally awarded grants and cooperative agreements. POs come from a range of backgrounds and are typically skilled in program management, and officers at the CDC often have had experience working at the state or local level. POs provide help and support to PIs and their program managers. Project managers often oversee the day-to-day operations of research grants and, as such, need to work with someone who can help them with work-plan development, budgets, and developing staffing requirements. However, owing to the unique and complex nature of the CORD project, it was decided that a team approach would be beneficial to this project given that it would bring specialized expertise for different project areas. Therefore, the CDC created a team consisting of a senior subject matter expert, scientific collaborator, behavioral scientist evaluator, and PO. Each of these individuals would help oversee the project for at least $25-50 \%$ of their workload.

Each of the CORD team members brought a specialized background to the CORD project and the team. The team includes two pediatricians with federal- and local-level public health experience and academic research training, a nurse with public health experience, and a PO with public health and health education experience. The roles of each CORD team member are described below.

The senior subject matter expert oversees the CORD team and coordinates the National Steering Committee (SC) of Federal Partners, which has an interest in the outcome of the project. The SC includes the Administration for Children and Families, the CDC, the Centers for Medicare and Medicaid Services, the Health Resources and Services Administration, and the National Institutes of Health. It is important for this person to be a subject matter expert in the obesity prevention and treatment areas to build partnerships with the SC and the demonstration sites. The SC meets with the senior subject matter expert on a regular quarterly schedule to stay connected with the activities of the project and provide input into the scientific methods, interventions chosen, the evaluation plan, the data collected, and other research issues.

The scientific collaborator serves as the main contact in working with the demonstration sites and the evaluation center researchers on the analytic design, sample selection, interventions, review of protocols, and strategies for building 
and strengthening the evaluation and design elements within and across the sites. Other activities include reviewing and providing input on research proposals, providing scientific and framework guidance for development of measures, and providing input across the evaluation plans, including impact, process, and sustainability.

The behavioral scientist evaluator helps the demonstration grantees and the evaluation center develop and implement their respective evaluation plans. This includes providing technical assistance to all aspects of the CORD evaluation, reviewing the individual grantee evaluation plans for the sites and the cross-site evaluation and plan, and providing technical expertise for grantee partners. Part of the evaluator's role was to help create a common set of measures that were used by all sites. This included negotiation of the different measures across all of the sites.

The PO's role is to act as the liaison between the sites, the CORD team members, and the grants administration office. This involves planning and scheduling monthly calls, coordinating activities, planning site visits, and gathering grantees together annually to promote sharing and discuss successes, lessons learned, and challenges posed by working across multiple sectors. The PO also provides technical assistance related to budgets, work plans, and evaluation plans. Ensuring the community context and community capacity, as well as linkages with local and state health departments and community coalitions, is also a critical lens that the PO brings to the project.

The CDC-CORD team approach to providing technical assistance is a robust model in several ways (Fig. 1). That is, the CDC-CORD team's approach:

1. Provides additional levels of support to the project with all members of the CORD team being involved in working with the grantees on a regular basis.

2. Assesses both management and scientific issues firsthand for each grantee during routine teleconferences and site visits.

3. Provides an opportunity to maintain institutional memory about the project in the event that one person leaves the CORD team before the cooperative agreement is complete.

4. Ensures that the appropriate level of staff can develop the most productive relationships when applicable (e.g., healthcare professionals with healthcare professionals, evaluators with evaluators, and program managers with POs).

\section{Program Management}

The CORD project team developed several procedures that were put into place to make CORD a successful research

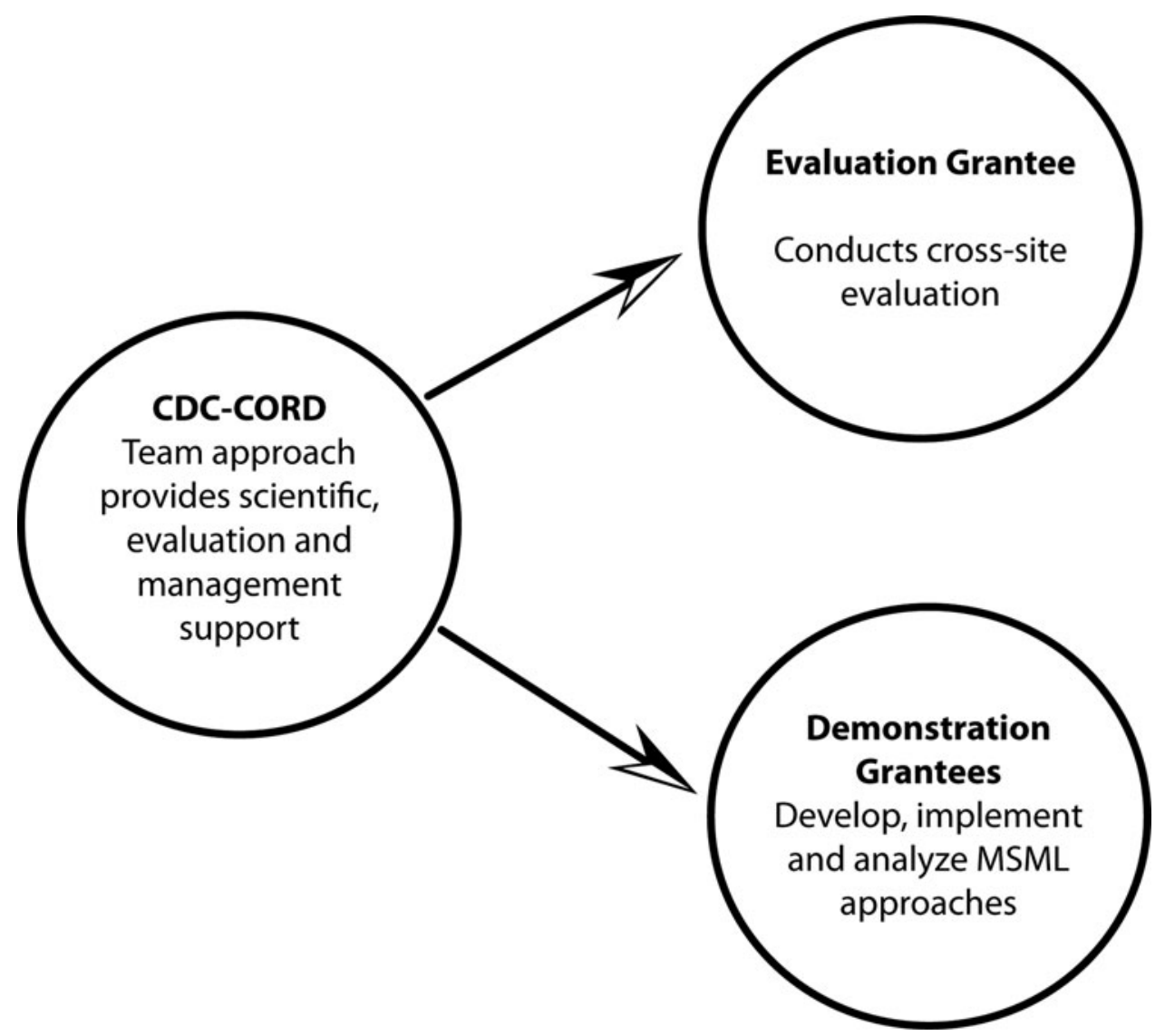

Figure 1. The Childhood Obesity Research Demonstration (CORD) project was designed to have a CDC-CORD team that provides scientific, evaluation, and management support to the grantees. The grantees include an evaluation grantee, which conducts an overall evaluation of the three unique multisector, multilevel (MSML) interventions being conducted by the demonstration site grantees. 
project. Before work began with the grantees, the CDCCORD team was involved in several preparatory projects to ensure that the team had the knowledge and background needed to provide technical assistance. This included reviewing existing interventions as the evidence base to draw from, creating a framework for the model, and creating common measure requirements for the grantees that facilitated the start of year 1 grant-planning activities.

Another important aspect was the establishment of a monthly call schedule with each grantee that included the whole CDC-CORD team, so that updates related to evaluation, data, and research questions could be discussed on each call. Twice-monthly calls were set up with all grantees to ensure information sharing. The grantees would decide on specific call topics for each conference call. For example, some of the topics included evaluation measures in select settings, enhancing recruitment, working with school administrators, and defining reach. The CORD team worked with subject matter experts across the CDC to provide additional guidance and resources to the grantees as necessary.

The team also helped establish protocols for site-specific and cross-site publications. The CDC-CORD team worked with the grantees in collaboration to establish a publication committee, a publication and presentation protocol, and a mechanism for keeping track of dissemination products, including presentations, publications, and posters.

Visits by the CORD team to each grantee were also planned yearly. At least two CORD team members attended each site visit to continue to develop the relationship with the different staff members. During the site visits, the CORD team meets with the different staff and community members to determine progress. The evaluation center also participated in the site visits. This allowed the evaluation center to identify the context of the communities in which the grantees were working and meet directly with the demonstration-site evaluation staff. Reverse site visits were planned to bring all of the grantees together at the CDC offices to meet with the demonstration site teams, the CDCCORD team, and other CDC subject matter experts. These meetings gave the grantees time to share their interventions, evaluation plans, and issues with the other grantees and develop a shared sense of accomplishments. These meetings also provided opportunities to problem solve issues and build collaboration and trust between the project sites.

The CORD team worked closely with the evaluation center to provide technical assistance as they developed their cross-site evaluation plan. The CDC-CORD team participated in weekly calls with the process, impact, and sustainability evaluation teams to help provide technical assistance on the cross-site evaluation development. Technical assistance was also provided to individual sites. The team also worked with the evaluation center to create the evaluation framework, advise on inclusion/exclusion criteria, review and enhance recruitment strategies, determine how to categorize information, and work collaboratively on reviewing the publications.

\section{Discussion}

The project is still in progress, but several important lessons have already been learned. The CDC-CORD team members needed to develop a close working relationship early in the process, so that they were able to explicitly describe the CDC-CORD team approach to the grantees. This was done by having retreats and weekly meetings to discuss updates, plan the next steps, and coordinate activities. It was also important that CDC-CORD team members ensure that all members of the team participate on a regular basis and dedicate adequate time to the project. It was also critical that all CDC-CORD team members play a role in planning meetings, conference calls, and site visits.

\section{Conclusion}

The CORD approach has worked to provide technical assistance to grantees in as efficient a manner as possible. At the end of CORD, formal feedback will be sought from the grantees to determine how this team approach worked and share this information with other programs that want to improve community health by building community-clinical linkages.

\section{Acknowledgments}

The authors acknowledge Suzianne Garner for her guidance and assistance in managing CDC-CORD and CDC advisors Allison Nihiser and Dr. Seraphine Pitt-Barnes.

\section{Author Disclosure Statement}

No competing financial interests exist. The findings and conclusions in this report are those of the authors and do not necessarily represent the official position of the CDC.

\section{References}

1. Ogden CL, Carroll MD, Kit BK, et al. Prevalence of childhood and adult obesity in the United States, 2011-2012. JAMA 2014;311:806-814.

2. Cunningham SA, Kramer MR, Narayan KM. Incidence of childhood obesity in the United States. N Engl J Med 2014;370:403-411.

3. Wang Y, Beydoun MA. The obesity epidemic in the United States - Gender, age, socioeconomic, racial/ethnic, and geographic characteristics: A systematic review and meta-regression analysis. Epidemiol Rev 2007;29:6-28.

4. Institute of Medicine. Accelerating Progress in Obesity Prevention: Solving the Weight of the Nation. The National Academies Press: Washington, DC, 2012.

5. Folta SC, Kuder JF, Goldberg JP, et al. Changes in diet and physical activity resulting from the Shape Up Somerville community intervention. BMC Pediatr 2013;13:157.

6. Story, M, Kaphingst DM, Robinson-O'Brien R, et al. Creating healthy food and eating environments: Policy and environmental approaches. Annu Rev Public Health 2008;29:253-272. 
7. Foltz JL, May AL, Belay B, et al. Population-level intervention strategies and examples for obesity prevention in children. Annu Rev Nutr 2012;32:391-415.

8. Waters E, de Silva-Sanigorski A, Hall BJ, et al. Interventions for preventing obesity in children. Cochrane Database Syst Rev 2011; (12):CD001871.

9. Dietz W, Lee J, Wechsler H, et al. Health plans' role in preventing overweight in children and adolescents. Health Aff (Millwood) 2007;26:430-440.

10. Centers for Disease Control and Prevention. Childhood Obesity Demonstration Project: Implementing strategies across the community to help families with childhood obesity. Available at www .cdc.gov/obesity/childhood/researchproject.html Last accessed July 17, 2014.

11. Dooyema, C, Belay MD, Foltz MD, et al. The Childhood Obesity Research Demonstration Project: A comprehensive community approach to reduce childhood obesity. Child Obes 2013;9:454459.

12. Disis M, Slattery JT. The road we must take: Multidisciplinary team science. Sci Transl Med 2010;2:1-4.
13. Post C, De Lia E, DiTomaso N, et al. Capitalizing on thought diversity for innovation. Res-Technol Manage 2009;52:14-25.

Address correspondence to:

Nancy Williams, MSPH

Project Officer

Obesity Prevention and Control Branch

Division of Nutrition, Physical Activity,

and Obesity

National Center for Chronic Disease

Prevention and Health Promotion

Centers for Disease Control and Prevention

4770 Buford Highway, F77

Atlanta, GA 30341

E-mail:ndw6@cdc.gov 